УДК 539.126 .4

\title{
Интерпретация данных об экстремальных погодных явлениях в рамках гипотезы о квазирезонансном механизме формирования блокирующих процессов
}

\author{
К.Ю. Суковатов, Н.Н. Безуглова
}

Институт водных и экологических проблем СО РАН (Барнаул, Россия)

\section{Data Interpretation for Weather Extremes on the Basis of Quasiresonance Hypothesis of Blocking Formation}

\author{
K.Yu. Sukovatov, N.N. Bezuglova \\ Institute for Water and Environmental Problems SB RAS (Barnaul, Russia)
}

В последние годы для теоретического исследования формирования меридиональных процессов определенного типа наряду с другими подходами используется гипотеза о квазирезонансном усилении волн Россби возмущениями циркуляции атмосферы, обусловленными термическими и орографическими неоднородностями подстилающей поверхности. В настоящей работе авторами проведена интерпретация данных реанализа и спутниковых данных в рамках гипотезы о квазирезонансном механизме формирования блокирующих процессов. На основе анализа параметров рассматриваемых волновых возмущений выделены ситуации, определяющие формирование условий, благоприятных для реализации механизма квазирезонансного усиления волнами Россби малоамплитудных колебаний атмосферной циркуляции. Построены пространственные распределения меридиональной компоненты скорости ветра в тропосфере, иллюстрирующие наблюдаемые закономерности. Для всех рассмотренных случаев условия реализации квазирезонансного усиления выполняются для компонент с зональными волновыми числами $k=6,7,8$. Для рассмотренных экстремальных погодных явлений значения анализируемых гармоник превышают 1.5-ное стандартное отклонение для средних климатологических значений соответствующих гармоник меридиональной скорости за период 1980-2011 гг.

Ключевые слова: меридиональные процессы, квазирезонанс, волны Россби, экстремальные погодные явления.
The quasiresonance hypothesis is used among other approximations for theoretical study of blocking formation in the recent years. It is propose the magnification of quasistationary waves resulted by thermal and orographic inhomogenities of underlying surface through quasiresonance. In this paper authors perform the interpretation of reanalysis and satellite data for weather extremes situations on the basis of quasiresonance hypothesis that describs mechanism of amplification for amplitudes of quasistationary waves. Base on the analysis of atmospheric waves parameters the situations favorable for realization of this mechanism were selected and described. The spatial distribution of the meridional velocity of atmospheric wind in troposphere was derived to illustrate all considered weather extreme situations. In all cases the conditions necessary for quasiresonance amplification are performed for wave modes with zonal wave numbers. All the considered weather extremes situations exhibit amplitudes for monthly Fourier components that are about 1.5 SD from the 1980-2011 monthly climatology data for the meridional velocity for the corresponding wave numbers.

Key words: meridional processes, quasiresonance, Rossby waves, weather extreames.

DOI 10.14258/izvasu(2018)4-06

•Работа выполнена в рамках проекта ФНИ РАН № 0383-2016-0005. 


\section{Введение}

В последние десятилетия происходящие климатические изменения могут быть охарактеризованы как процессы усиления потепления в полярной области и увеличения повторяемости меридиональных процессов, блокирующих западно-восточный перенос в средних широтах северного полушария [1-5]. Существует ряд научных гипотез, объясняющих усиление потепления в полярной области, увеличение повторяемости меридиональных процессов в средних широтах, а также возможную взаимосвязь между указанными явлениями [1-5]. Однако ни одна из этих гипотез не может в полной мере объяснить все наблюдаемые процессы. В последние годы для теоретического исследования формирования меридиональных процессов определенного типа наряду с другими подходами $[1,2]$ используется гипотеза о квазирезонансном усилении волн Россби возмущениями циркуляции атмосферы, обусловленными термическими и орографическими неоднородностями подстилающей поверхности $[6,7]$. Квазирезонансное взаимодействие гармоник рассматриваемых атмосферных волн приводит к существенному увеличению амплитуд квазипериодических волновых структур и блокированию западно-восточного переноса на временных масштабах свыше недели. Указанные процессы приводят к реализации экстремальных погодных условий в отдельных районах: слабого/сильного увлажнения, возникновения засух/наводнений, усиления эрозионных процессов [8].

В настоящей работе авторами проведена интерпретация данных реанализа и спутниковых данных в рамках гипотезы о квазирезонансном механизме формирования блокирующих процессов. На основе анализа параметров рассматриваемых волновых возмущений выделены ситуации, в которых реализуется механизм квазирезонансного усиления волнами Россби малоамплитудных колебаний атмосферной циркуляции. Построены пространственные распределения меридиональной компоненты скорости ветра в тропосфере, иллюстрирующие наблюдаемые закономерности.

\section{1. Используемые методы}

Крупномасштабная атмосферная циркуляция в средних широтах в основном характеризуется бе- гущими свободными волнами Россби синоптического масштаба с зональными волновыми числами $k$, распространяющимися в долготном направлении, и квазистационарными планетарными волнами Россби с различными значениями зональных волновых чисел $m$, которые возникают как отклик атмосферной циркуляции на воздействие квазистационарных возмущений, связанных с наличием термических и орографических неоднородностей подстилающей поверхности [9]. Квазистационарная компонента свободных волн Россби синоптического масштаба обычно слаба (имеет малые амплитуды 1.5-2.0 м/с). В условиях реализации квазирезонансного механизма отдельные моды захваченных волн Россби синоптического масштаба усиливают обычно слабый малоамплитудный отклик атмосферной циркуляции на термические и орографические неоднородности подстилающей поверхности, создавая высокоамплитудные (4.0-5.0 м/с) атмосферные паттерны (барические системы), блокирующие западно-восточный перенос.

Для теоретического исследования этого процесса необходимо решить следующие задачи: провести теоретический анализ выполнения условий возникновения широтного захвата волн Россби синоптического масштаба и их квазирезонансного усиления квазистационарными модами планетарных волн Россби; на основе решения уравнения потенциального вихря в квазигеострофическом приближении для баротропной атмосферы рассчитать широтное распределение меридионального волнового числа квазипериодических возмущений, а также его зависимость от среднего поля зональной скорости ветра, определить широтное положение точек поворота, ограничивающих условный «атмосферный волновод»; провести расчет амплитуд волновых атмосферных паттернов, блокирующих западно-восточный перенос.

Для решения поставленных задач использовалось линеаризованное, нестационарное, бездивергентное уравнение сохранения потенциального вихря на сфере для эквивалентного баротропного уровня (ЭБУ) [9]. Предполагая, что ЭБУ ограничен диапазоном высот 500-300 гПа, запишем уравнение сохранения потенциального вихря в квазигеострофическом приближении:

$$
\begin{aligned}
& \left(\frac{\partial}{\partial t}+\alpha \frac{\partial}{\partial \lambda}\right) \Delta \psi+\left(2 \Omega-\frac{1}{a \cos (\phi)} \Delta \bar{u}\right) \frac{\partial \psi}{\partial \lambda}=\frac{2 \Omega \alpha a^{2}}{\tilde{T}} \sin (\phi) \frac{\partial T}{\partial \lambda}- \\
& -\frac{2 \Omega \sin (\phi) \alpha_{o r} a^{2}}{H} \frac{\partial h_{o r}}{\partial \lambda}-k_{h} \frac{\Delta \psi}{a^{2}}-k_{z} \frac{\Delta \psi}{H^{2}},
\end{aligned}
$$

$t$ - время, $\lambda$ - долгота, $\phi$ - широта, $\Omega$ - угловая скорость вращения Земли, $a-$ радиус Земли, $\tilde{T}-$ средняя температура в атмосферном слое, $\Delta-$ горизон- тальный оператор Лапласа, $\alpha=\bar{u} / a \cdot \cos (\phi)-$ индекс атмосферной циркуляции, $\bar{u}-$ усредненная по атмосферному слою широтная компонента скорости ветра, 
$h_{o r}$ - масштаб орографической неоднородности, $\alpha_{o r}=\bar{u}_{o r} / a \cdot \cos (\phi)$ - индекс циркуляции, характеризующий отклик циркуляции на орографическую неоднородность, $\bar{u}_{o r}$ - возмущение широтной компоненты скорости ветра, $\bar{h}_{o r}$ - широтно усредненное значение масштаба орографических неоднородностей, $\psi-$ функция тока для атмосферного слоя, $T-$ температура в атмосферном слое, $k_{h}$ - горизонтальная компонента коэффициента турбулентного обмена, $k_{z}$ - вертикальная компонента коэффициен- та турбулентного обмена, $H$ - масштаб высоты атмосферного слоя. На начальном этапе необходимо рассчитать широтное распределение меридионального волнового числа для квазистационарных свободных плоских волн Россби, используя уравнение, записанное в ВКБ-приближении для стационарного решения уравнения (1) с нулевой правой частью. Тогда мы получим соотношение, связывающее квадрат меридионального волнового числа со средней зональной (широтной) скоростью атмосферной циркуляции.

$$
l^{2}=\frac{2 \Omega \cos ^{3}(\phi)}{a \bar{u}}-\frac{\cos ^{2}(\phi)}{a^{2} \bar{u}} \frac{d^{2} \bar{u}}{d \phi^{2}}+\frac{\sin (\phi) \cos (\phi)}{a^{2} \bar{u}} \frac{d \bar{u}}{d \phi}+\frac{1}{a^{2}}-\left(\frac{k}{a}\right)^{2},
$$

где $k=K a, k-$ безразмерное зональное волновое число.

Квадрат волнового числа может менять знак на некоторых широтах, называемых точками поворота (средняя скорость $\bar{u}$ в этих точках равна нулю). Согласно $[10,11]$ волновая энергия свободных волн Россби синоптического масштаба испытывает сильное снижение в критической точке, в точке поворота происходит сильное отражение волны. Уравнение (2) «не работает» в непосредственной близости от точек поворота, и широтная зависимость энергии квазистационарной волны Россби описывается в этой области как квадрат функции Эйри $[6,7,11]$. В ситуациях, когда присутствуют две точки поворота, отдельные гар- моники синоптических волн Россби с зональными волновыми числами $k=m$ могут трансформироваться в высокоамплитудную волну, захваченную в широтном волноводе, ограниченном точками поворота. Эта ситуация реализуется при выполнении условий: 1) $\bar{u}>0, l^{2}>0$ внутри волновода, $\bar{u}>0, l^{2}>0$ вблизи границ волновода; 2) $\left|d l^{-1} / a d \phi\right|<1$ внутри волновода; 3) общая ширина широтного волновода определяется характерным масштабом соответствующей функции Эйри $[6,7]$. Обычно решение стационарного варианта уравнения (1) имеет малоамплитудные решения, но при выполнении условий 1-3 получаются высокоамплитудные решения. Искомая величина амплитуды определяется соотношением

$$
\tilde{A}_{m}=\tilde{A}_{m}^{\text {ort }} \frac{2 \Omega \sin \left(\phi_{m, 0}\right) \cos ^{2}\left(\phi_{m, 0}\right)}{\left\{\left[K^{2}-M^{2}\right]^{2}+\left(L / a^{2}+R o^{2} / L\right) M^{2}\right\}^{1 / 2}},
$$

где $K=k / a, M=m / a, L-$ радиус Россби, Ro- число Россби.

2. Исследуемая территория и используемые данные

Исследуемая территория представляет собой прямоугольную область, ограниченную координатами 40-65 с.ш., 50-90 в.д. В работе использовались данные реанализа MERRA2 и спутниковые данные, полученные с помощью мультиплатформенной системы усвоения данных GIOVANNI (https:/giovanni.gsfc. nasa.gov/giovanni/) для доступа к данным из разных источников: сеточные массивы данных, спутниковые данные, данные реанализа, результаты модельных расчетов и т.д. Также использовались данные реанализа NCEP-DOE Reanalysis 2, полученные с серверов NOAA (https://www.esrl.noaa.gov/psd/data/gridded/data. ncep.reanalysis $2 . \mathrm{html}$ ).

Анализировались средние суточные и месячные значения меридиональной компоненты скорости ветра в тропосфере для барических уровней 300 и 500 гПа, являющихся границами эквивалентного баротропного уровня (ЭБУ). Для оценки изменения меридиональной компоненты атмосферной циркуляции в периоды наблюдений отдельных экстремальных явлений (засухи в Западной Сибири 1991, 2004 и 2012 гг., наводнение в Западной Сибири 2014 г.), а также для построения ее пространственных распределений использованы среднесуточные значения.

Средние месячные значения характеристик меридиональной циркуляции для летних месяцев указанных лет использовались для сравнения с климатологическими средними за 1980-2011 гг. (т.н. 30-летний период глобального потепления).

\section{3. Анализ полученных результатов}

Анализ широтного распределения меридиональных волновых чисел квазипериодических возмущений и их зависимости от среднего поля зональной составляющей скорости ветра проводились с использованием аналитического решения стационарного линеаризованного уравнения сохранения потенциального вихря в рамках ВКБ-приближения. Далее на основании полученного решения были рассчитаны амплитуды квазипериодических волновых паттернов, блокирующих западно-восточный перенос. 
Интерпретация данных об экстремапьных погодных явпениях...

В качестве входных данных использовались спутниковые данные и данные реанализа (https:/giovanni. gsfc.nasa.gov/giovanni/, https://www.esrl.noaa.gov/psd/ data/gridded/data.ncep.reanalysis2.html).

На основе анализа широтного распределения меридионального волнового числа и его зависимости от средней зональной скорости ветра были выделены ситуации, где поле ветра в среднеширотной тропосфере изменяется таким образом, что формируются две точки поворота, которые могут выступать в качестве границ области условного «широтного волновода», в пределах которого происходит квазирезонансное усиление исследуемых волн. Для всех рассмотренных случаев условия реализации квазирезонансного усиления выполняются для компонент с зональными волновыми числами $k=6,7,8$. В результате селекции данных для ситуаций, соответствующих условиям реализации квазирезонансного усиления, были построены пространственные распределения меридиональной компоненты скорости ветра.

На рисунке приведено пространственное распределение меридиональной компоненты скорости ветра $V$ в тропосфере для уровня 300 ГПа. Указанное пространственное распределение соответствует ситуации второй паводковой волны, усиленной атмосферными осадками в конце мая - начале июня 2014 г. Для построения этого распределения использовались данные реанализа NCEP-DOE Reanalysis 2 о средних суточных значениях меридиональной компоненты скорости ветра в тропосфере. Полученные распределения представляют собой высокоамплитудные атмосферные паттерны, блокирующие западно-восточный перенос. Их формирование, по-видимому, обусловлено существованием высокоамплитудных гармоник волн Россби с зональными волновыми числами $k=6,7,8$. В разные годы для отдельных месяцев может преобладать какая-то одна мода. Например, во время «тепловой волны» 2010 г. в европейской части России наиболее сильный вклад вносила гармоника с зональным волновым числом $k=m=6$ [6]. Рассмотренные метеорологические ситуации характеризуются рекордными для данного региона значениями приземной температуры воздуха, количества атмосферных осадков и других гидрометеорологических параметров.

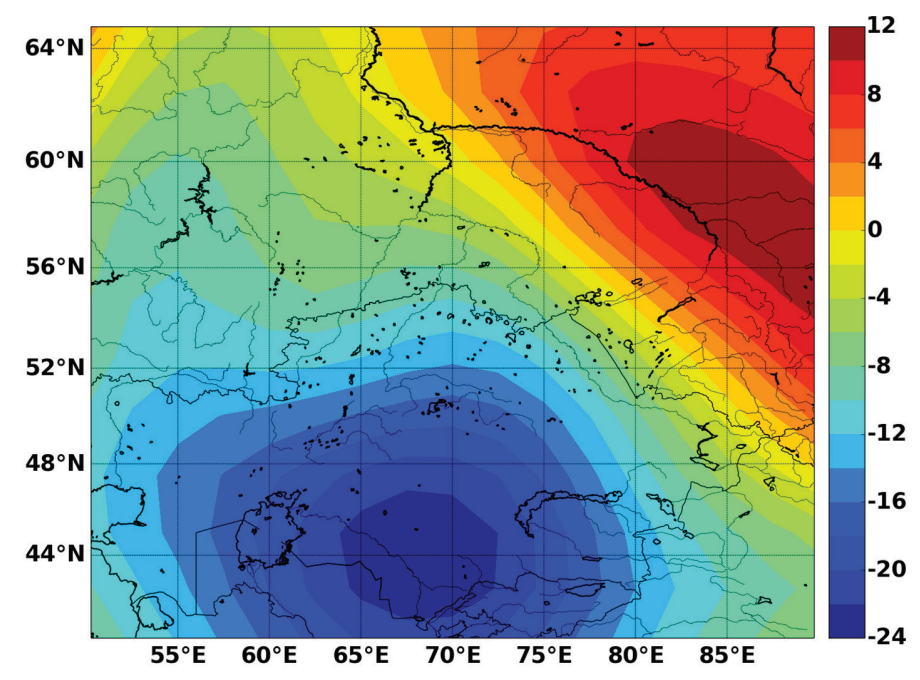

Рис. Пространственное распределение меридиональной компоненты скорости ветра $V$ в тропосфере для уровня 300 ГПа (28.05.14-3.06.14). Значения $V$ приведены в м/с

Аналогичным образом по массивам данных реанализа и спутниковых данных были рассчитаны средние месячные значения меридиональной компоненты скорости ветра и среднемесячные значения для Фурье-гармоник с соответствующими зональными волновыми числами. Для рассмотренных экстремальных погодных явлений значения анализируемых гармоник с зональными волновыми числами $k=m=6,7,8$ превышают 1,5-ное стандартное отклонение для средних климатологических значений соответствующих гармоник меридиональной скорости за период 1980-2011 гг.

\section{Заключение}

Рассчитаны параметры волновых возмущений, участвующих в квазирезонансном усилении квазистационарных волн Россби и, предположительно, приводящих к формированию меридиональных процессов, блокирующих западно-восточный перенос в тропосфере средних широт северного полушария. Сделан вывод о том, что гармоники волн Россби с зональными волновыми числами $k=m=6,7,8$ в результате квазирезонанса испытывают существенное увеличение амплитуды, а указанный процесс может приводить к формированию высокоамплитудных меридиональных паттернов и, как следствие, реализации экстре- 
мальных погодных явлений в умеренных широтах северного полушария. Для ситуаций, в которых реализуются условия формирования исследуемых блокирующих процессов, построены пространственные распределения меридиональной компоненты скорости ветра в среднеширотной тропосфере. Рассчитаны среднемесячные значения анализируемых параметров. Сделан вывод о превышении значений анализируемых гармоник 1,5-кратного стандартного отклонения для средних климатологических значений соответствующих гармоник меридиональной скорости за период 1980-2011 г.

\section{Библиографический список}

1. Francis J. A., Vavrus Stephen J. Evidence for wavier jet stream in response to rapid Arctic warming // Environmental Research Letters. — 2015. — V. 10: 014005. DOI:10.1088/17489326/10/1/014005

2. Francis J.A., Skific N. Evidence linking rapid Arctic warming to mid-latitude weather patterns // Phil. Trans. A Math Phys Eng Sci. - 2015. - V. 373 (2045): 20140170. DOI:10.1098/rsta.2014.0170

3. Barnes E.A. Revisiting the evidence linking Arctic amplification to extreme weather in midlatitudes // Geophysical research letters. - 2013. - V. 40. DOI:10.1002/grl.50880

4. Screen J.A., Simmond I. Amplified mid-latitude planetary waves favour particular regional weather extrems. Nat. Clim. Change. - 2014. - V. 4. DOI:10.1038/nclimate2271

5. Cohen J.L., Screen J.A., Furtado J.C., Barlow M., Whittleston D., Coumou D., Francis J.A., Dethloff K., Entekhabi D., Overlandand J.E., Jones J. Resent Arctic amplification and extreme mid-latitude weather. Nat. Geosci. - 2014 - V. 7. DOI:10.1038/NGEO2234
6. Petoukhov V. et al. Quasiresonant amplification of planetary waves and recent Northern Hemisphere weather extremes // Proc. Natl. Acad. Sci. USA. - 2013. - V. 110. DOI:10.1073/pnas.1222000110

7. Kornhuber K. et al. Detection of quasiresonant amplification of planetary waves and their connection to northern hemisphere summer heat extremes EGU General Assembly Conference Abstracts. - 2014. -V. 16: 15164.

8. Мохов И. И. Российские исследования в области атмосферных наук и метеорологии в 2011-2014 гг. // Известия Российской академии наук. Физика атмосферы и океана. - 2016. - Т. 52, № 2.

9. Педлоски Дж. Геофизическая гидродинамика. T. 1, 2. - M., 1984.

10. Charney J. G., and Drazin P. G. Propagation of planetaryscale disturbances from the lower into the upper atmosphere. J. Geophy. Res. - 1961. - V. 66.

11. Dickinson R.E. Development of a Rossby wave critical level. J. Atmos. Sci. - 1970. - V. 27. 